\title{
New tools in studies on iron nutrition Principles, applications and consequences
}

\author{
By Leif Hallberg
}

\begin{abstract}
New tools to study iron nutrition are reviewed in this article. An algorithm is devised to predict dietary iron absorption from meals and diets based on contents of dietary factors influencing heme and nonheme iron absorption. Sum of iron absorption from single meals was found to equal total dietary iron absorption. Observed relations between iron absorption, iron requirements and iron stores can be described as an exponential equation allowing calculations of any stationary state. After integration of the equation, rate of changes in iron stores, or hemoglobin iron deficits, can be calculated when any factor is modified. Control of dietary iron absorption is extremely efficient, effectively preventing iron overload in all who are not genetically predisposed. Suggested relations between iron stores and disease may rather be independent relations between diseases and serum ferritin. Key words: control of iron absorption, iron requirements, iron stores prediction of iron absorption, single meal
\end{abstract}

\section{Introduction}

Iron nutrition in a population is determined by the individual variation in iron requirements and the corresponding variation in dietary iron absorption. Over the years methods have been developed to measure the absorption of iron from foods, meals and lately whole diets. Similarily, our knowledge has grown about the amounts of iron required to be absorbed by different population groups to cover the needs for growth and for losses of iron from the body.

Much is known about the effect of different dietary and other factors on iron absorption but it has not been possible to predict the amounts of iron expected to be absorbed from composite meals or the diet as a whole. Similarily, it has not been possible to estimate the expected effects of variations in dietary iron bioavailability or iron requirements on iron status, expressed as amounts of stored iron or as amounts of hemoglobin iron deficits.

Leif Hallberg, $\mathrm{MD}, \mathrm{PhD}$. Prof. emeritus. Internal Medicin, Göteborg University, Dept of Clinical Nutrition, Sahlgrenska University Hospital. SE-413 45, Göteborg, Sweden. E-mail: nutrition@clinnutr. gu.se or hallberg@medfak.gu.se

Leif Hallberg received the second Danone International Prize for Nutrition in 1999 , for his significant contributution to the knowledge of nutrition in the field of hematology and iron metabolism.

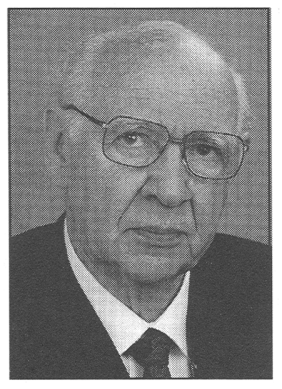

Recently, an algorithm has been developed to predict the amounts of iron absorbed from meals and diets with different composition (1). At about the same time methods have also been developed to estimate the effects of variations in iron requirements and dietary iron bioavailability on iron status $(2,3)$. The present paper shortly reviews these new tools and their practical applications

\section{Dietary iron absorption and requirements}

Today it is possible to determine the amounts of heme and nonheme iron absorbed from a specific food using the extrinsic radioiron technique. This method uses two different radioiron tracers $\left({ }^{59} \mathrm{Fe}\right.$ and $\left.{ }^{55} \mathrm{Fe}\right)$ to label the two kinds of dietary iron, one for heme iron (as biosynthetically radioiron labelled hemoglobin) and one for nonheme iron (as an inorganic iron salt). From knowledge about the contents of these two kinds of iron in a meal and the absorption of the two corresponding tracers, it is possible to calculate their absorption. This basal methodology was developed about 30 years ago (4) and has been confirmed and carefully validated by several investigators (5-9). This extrinsic tag method has also been extensively used in absorption studies on other minerals (e.g. zinc, manganese and calcium), as we early suggested.

Several studies using the extrinsic tag method were made to identify and describe the effects of various factors influencing the absorption of nonheme iron (e.g. ascorbic acid, meat, fish and seafoods, alcohol, phytate and other inositol phosphates, iron-binding polyphenols, calcium, eggs and soy protein) and to describe the relationship between iron status and food iron absorption (10-29) Several studies were also made to measure nonheme iron absorption from composite meals and to examine the interaction between different factors. (Reviews: $(8,24)$ ).

More recently iron absorption from the whole diet was measured by separately labelling heme and nonheme iron using two different radioiron isotopes. To ensure the same specific activity of the nonheme iron, in all meals under a certain study period, for example, 5 to 10 days as used in previous studies all meals were separately labelled (30-32). Similarily, heme iron in all meals was also homogenously labelled to an identical specific activity. The total absorption of ${ }^{59} \mathrm{Fe}$ labelled foods was measured from the total retention of the tracer two weeks after study completion, using a whole body counter. The total absorption of ${ }^{55} \mathrm{Fe}$ labelled foods was determined by analysing the ratio of ${ }^{55} \mathrm{Fe}$ and ${ }^{59} \mathrm{Fe}$ in a blood sample.

Iron requirements and their variation have also been extensively studied. The basal losses of iron from skin and different mucosal surfaces was examined based on the rate of dilution over several years of the long-lived radioiron isotope ${ }^{55} \mathrm{Fe}$ in healthy men (33). Menstrual iron losses could be validly measured using a specifically developed technique to ensure complete collection and analyses of menstrual blood losses. These losses were found to be very constant in the individual woman but varied markedly between different women (34). The iron requirements and their variation could thus be measured in random samples of women $(35,36)$. Sweat iron losses, once thought to be considerable, were found to be neg- 
ligible and possible to disregard in iron balance considerations (37). Growth requirements in infancy, childhood and adolescence have been assessed by factorial analysis $(38,39)$.

The purpose of the present review is to briefly present new methodological developments and their practical use and implications.

\section{A new algorithm to predict the absorption of dietary iron}

Over the years several attempts have been made to develop an algorithm to predict the amount of iron expected to be absorbed from a meal. We presented the first algorithm already in 1978 (40). It had mainly a didactic purpose aimed at illustrating the importance of considering different dietary factors in the absorption of dietary iron. Only factors enhancing iron absorption, such as meat and ascorbic acid, were considered in this first algorithm. Several attempts were made to improve the algorithm but no validation was made (41-43). Recently, a new algorithm has been developed and validated (1) to predict the amounts of iron absorbed from meals and diets based on all presently known factors, influencing the bioavailability of dietary iron. In Equation 2 in the algorithm (1) the Technical Editor of AJCN has incorrectly inserted two parenthesis. The correct Equation 2 should be: Absorption ratio $=1+0.01 A A+\log (P P+1)^{*}$ $0.01 * 10^{0.8875 * \log (A A+1)}$

where $\mathrm{AA}=$ ascorbic acid $(\mathrm{mg})$ and $\mathrm{PP}=$ phytate phosphorus (mg).

The new algorithm was based on the observation that in borderline iron replete subjects the absorption of iron from wheat rolls, made from low extraction flour and fermented to eliminate all detectable phytate, was very constant at a level of $22.1 \pm$ $0.18 \%$ in several series of studies $(\mathrm{N}=310)$ (1). These wheat rolls thus did not contain any known inhibitors or enhancers of iron absorption. When the wheat rolls were served with different factors that might influence iron absorption, such as ascorbic acid, it was possible to quantitatively compare the effect of different factors on iron absorption. To allow for variations in iron status, absorption figures were adjusted to a specific iron status, corresponding to a reference dose absorption of $40 \%$. Individual dietary factors suspected to influence iron absorption were thus examined when given in different amounts together with these "neutral" wheat rolls. For each factor, an equation describing the dose-effect relation was developed usually from exponential functions. The total effect of different factors known to influence nonheme iron absorption from a meal was then analysed by multiplying
$22.1 \%$ by all the enhancing and inhibiting factors present in a meal.

The interaction between different factors was also analyzed and included in the final equation to predict nonheme iron absorption from a meal. For example, the relative effect of ascorbic acid was more pronounced in the presence of phytate (inositol phosphates) and/or iron-binding polyphenols. Likewise, the relative enhancing effect of meat was more pronounced in the presence of iron-binding polyphenols and/or phytate (inositol phosphates). In the present algorithm, eight different dietary factors were considered: ascorbic acid, meat (including fish, other seafood and poultry), inositol phosphates (phytate), iron-binding polyphenols, calcium, soy protein, alcohol, and eggs. The data used to derive the equations originated mainly from studies in our laboratory but whenever possible to translate results from studies in other laboratories they were also included.

Heme iron absorption was calculated separately. The different factors mentioned for nonheme iron did not influence heme iron absorption with the exception of calcium. The same factor for calcium was used for heme and nonheme iron. The total amount of iron predicted to be absorbed from a meal was thus the sum of the absorption of nonheme and heme iron.

The validity of the present method to predict nonheme iron absorption from a meal was examined by comparing the observed mean absorption in each of 24 different meals served to groups of subjects (each including about 10 subjects) with the predicted absorption, as calculated from the present algorithm. The observed $\mathrm{r}^{2}$ value of the relationship (predicted/observed) was $0.987 ; \mathrm{N}=243$.

The algorithm can also be used to predict the absorption of iron from the whole diet based on the sum of the absorption of heme and nonheme iron in all the different single meals included in the diet over a certain period of time using whole body counting, as described above. Algorithm validity was examined by calculating the sum of the absorption of heme and nonheme iron from 20 different meals served to each of 31 subjects over 5 days (620 meals), using the algorithm and comparing the total calculated absorption figures with the total retention of separately and homogenously labelled heme and nonheme iron. The agreement obtained between observed and calculated absorption of heme and nonheme iron was very close. The mean difference in the 31 subjects was $<1 \%$.

It has been suggested that the variation in iron absorption from single meals under laboratory conditions might exaggerate the variation from the whole diet (42). It is certainly true that in a group of subjects the variation in iron absorption between single meals is far greater than the variation in total absorption from several meals (the diet). This obvious fact, however, does not invalidate the use of results of studies on single meals or the sum of the absorption from single meals to estimate absorption from the whole diet. The validity of the latter procedure was clearly established in our previous study. Studies on the potential effect on iron absorption due to interaction between sequential meals were also reported and discussed in our previous paper (1).

The algorithm to predict dietary iron absorption has several practical applications, such as evaluating the nutritional value of different meals with respect to iron (e.g. in school lunch programs, catering programs for the elderly), translating data from dietary surveys into amounts of iron expected to be absorbed, and evaluating the bioavailability of weaning foods with different compositions. The effect of different meal patterns, vegetarian and vegan meals, low meat diets etc can now be quantitatively compared and evaluated. Important applications of the algorithm are studies on effects expected on iron status by realistic dietary modifications in both developing and developed countries. More examples are presented in the original publication (1).

It was evident from the studies used in developing the algorithm that it is essential to know the detailed composition of meals and its variation over representative periods of time. Detailed knowledge about the chemical composition of meals has turned out to be essential in the practical use of the algorithm. There are several foods that have sufficient amounts of inhibitors or enhancers of nonheme iron absorption which need to be considered in the predictions. It should also be realized that there are common foods such as potatoes which have a low concentration of phytate but which still provide a considerable amount of phytate due to the total amount of potatoes often consumed in a meal. An appendix containing data about the contents of phytate and iron-binding polyphenols in some common foods is provided in the original publication to facilitate the immediate use of the algorithm (1).

In developing countries there is a lack of information about the chemical composition of local foods, spices and condiments which may contain, e.g., appreciable amounts of phytate and iron-binding polyphenols. By applying the algorithm, however, it is possible to quantitatively evaluate the potential effect of dietary modifications that are possible and thus determine what is acceptable and thus realistic. 


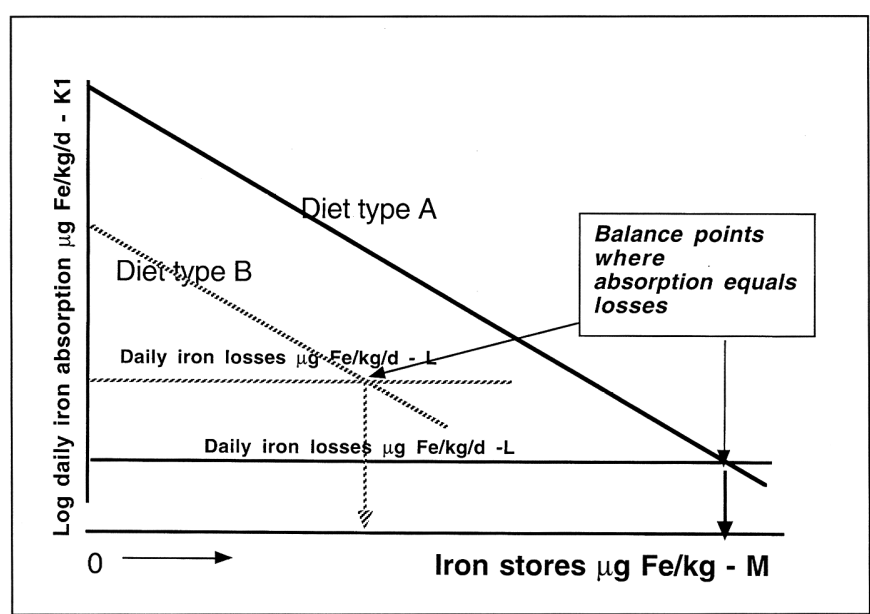

Figure 1. For explanations see text.

\section{Calculations of iron status - diet and iron requirements}

Several studies have shown a straight line relationship between iron absorption from so-called reference doses of inorganic iron $\left(3 \mathrm{mg} \mathrm{Fe}{ }^{++}\right)$and $\log$ serum ferritin $(26,44-$ 46). Newly established relationships between log iron absorption from whole diets and $\log$ serum ferritin (log SF) (30-32) have given a new meaning and dimension to the relationship between iron absorption and $\log$ SF. Moreover, a reevaluation of the relationship between log SF and iron stores has allowed valid calculations of dietary iron absorption in relation to amounts of stored iron under different conditions (2).

For three different diets we found straight line and parallell relationships between log iron absorption $(\mu \mathrm{gFe} / \mathrm{kg}$ b.w./d) and iron stores $(\mu \mathrm{gFe} / \mathrm{kg}$ b.w.). Diets with different bioavailability of its iron formed parallell regression lines (2). It is important to emphasize that all calculations are based on observations and that no model assumptions are required.

Figure 1 is a didactic illustration of the relationship between log dietary iron absorption (the product of bioavailability and iron intake) and iron stores (the solid oblique line). With increasing iron stores less iron is absorbed. The amount of iron needed to be absorbed to cover iron requirements is graphed as a solid horizontal line. At a certain point on the regression line the amount of iron absorbed intersects the horizontal line expressing the amount of iron required. Iron absorption thus equals the losses of iron at this point where a stationary state is reached and where thus iron stores can not increase any further. This stationary state can simply be calculated using the following equation: $k_{1} e^{-k 2} M=L$

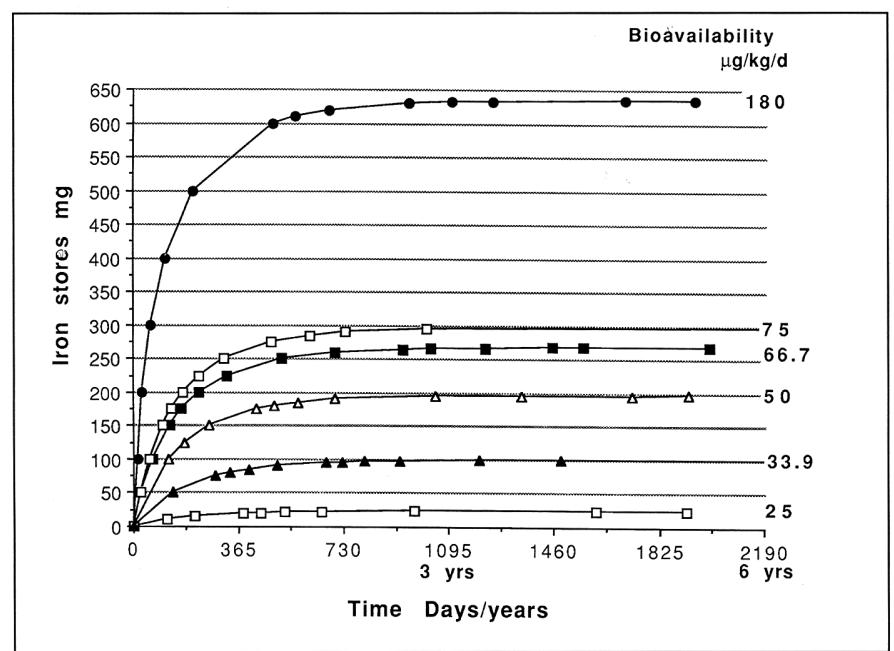

Figure 2. The increase in iron stores in menstruating women with median iron requirements and with diets of different bioavailability. The highest bioavailability $180 \mu \mathrm{gFe} / \mathrm{kg} / \mathrm{d}$ is an estimation of the bioavailability of the diet in prehistoric women with a very high intake of meat and ascorbic acid. The "75 diet" is a hypothetical, very high bioavailable diet in a present day woman. The " 66.7 " and the " 50 " diets are realistic diets with high bioavailability with low content of phytate, tannin and a high meat intake - two meals a day. The " 33.9 " is a common diet in women containing both meat and fish. The " 25 " is a mixed diet but with lower intake of meat and fish and a higher intake of milk products and cereals with a higher intake of phytate.

where $\mathrm{k}_{1}$ is the daily amount of iron $\mathrm{ab}$ sorbed from a certain diet at zero iron stores, $\mathrm{k}_{2}$ is a constant expressing the observed relationship between log iron absorption and iron stores (slope) which has been found to be the same for different diets $\left(\mathrm{k}_{2}=0.00024\right), \mathrm{M}$ is the amount of iron in stores and $\mathrm{L}$ the daily amount of iron lost. $\mathrm{L}$ and $\mathrm{k}_{1}$ are expressed as $\mu \mathrm{gFe} /$ $\mathrm{kg} / \mathrm{d}$ and $\mathrm{M}$ as $\mu \mathrm{gFe} / \mathrm{kg}$.

$\mathrm{k}_{1}$ is the product of dietary iron content and the traditional concept relative bioavailability, i.e. the fraction of iron absorbed at zero iron stores. In the original text $\mathrm{k}_{1}$ is just called bioavailability, for the sake of simplicity.

In the graph, another example is also inserted (as a hatched line): a diet with a lower bioavailability, $\mathrm{k}_{1}$, and a different loss value, $\mathrm{L}$, with its corresponding horizontal line. A different point of intersection will thus be obtained with its projected lower $\mathrm{M}$ value, iron stores, on the $\mathrm{x}$-axis.

The bioavailability of iron in an actual diet, $\mathrm{k}_{1}$, can be calculated by using the algorithm above and the composition of the meals included in the diet. With the known distribution of iron losses in women, for example (36), it is then possible to calculate the distribution of iron stores in women consuming known diets. Such calculations are necessary, for example, in evaluating the iron balance situation in pregnant women having different habitual diets. The iron balance in women using different contraceptive methods can also easily be calculated from the fact that contraceptive pills reduce menstrual losses by half, whereas the common intrauterine devices doubles the losses.

We have no experimental data describing the relationships between iron absorption, iron requirements and iron stores in infants and children. It is probable, however, that the strong relationships observed in adult men and women are based on some fundamental common biological mechanisms in iron metabolism which are very probably valid at all ages. Therefore, it is hard to believe that the relationships in adults between iron absorption, actual iron requirements and iron status would be different from those in infants and children, at least at ages above 6 months. However, before drawing these conclusion, further studies in children are undoubtedly needed.

By integration of the equation describing the stationary states it is possible to calculate the time needed to change iron stores from one amount to another by changes of iron losses (requirements) and/ or bioavailability (and/or amount) of dietary iron. The most unexpected observation, made early on, was that for all diets and all iron requirements during a period 
of positive iron balance there was an initial "rapid" growth of iron stores over slightly more than one year followed by an asymptotical, almost constant amount of stored iron already after 2-3 years. Actually, such a "rapid" first phase occurred both when inducing a positive and a negative iron balance (Figure 2.)

Our observations are consistent with observations that iron stores, by and large, remain constant in men after about 20 years of age. A strong evidence for almost constant iron stores in adult men is based on observations of a constant liver iron content at different ages. This was noted in extensive autopsy studies in subjects with traumatic death $(47,48)$. Moreover, in one of the studies, it was shown that in women in the fertile age period in USA, about $40 \%$ had very low liver iron concentrations indicating iron deficiency (48). This is of great interest considering the much lower prevalence figures, reported in the NHANES II studies made at about the same time as the autopsy studies, but using indirect measures of iron status and multiple diagnostic criteria for iron deficiency (49). A considerable underestimation of the prevalence of iron deficiency is expected when using multiple criteria (50). This fact has been emphasized in a previous study on methods for screening for iron deficiency where the validity of different diagnostic criteria were independently evaluated by stained bone marrow smears (50).

Another important implication of the present calculations of iron stores is the very efficient control of iron absorption leading to constant iron stores when dietary intake of iron and losses of iron are constant. In practice this means that it is impossible to develop an iron overload by consuming diets with even a very high content or high bioavailability of dietary iron, for example, due to a very high meat intake or due to a high level of iron fortification of foods with iron compounds of high bioavailability even when given in great amounts to subjects (e.g. men) with low iron requirements. The only exemptions would be (1) the rather few subjects who are homozygots for hereditary hemochromatosis (about 1/1000 in our estimations of the prevalence in the general population $(51,52)$ ) and (2) the even fewer subjects (in Scandinavia) with thalassemia major.

Teleologically, it may be reasonable to assume, that in early man consuming a diet expected to have a very high bioavailability of dietary iron due to a high meat intake (2), the risk of iron deficiency was low and that the theoretical risk of iron overload was non-existent. It is possible that effective mechanisms to prevent iron overload were essential for survival and that this is true in the whole animal kingdom.

The extensive NHANES II studies showed that a considerable fraction of especially elderly people have high levels of serum ferritin suggesting high iron stores (49). No direct data, however, support this interpretation. A translation of SF into iron stores must be made with great caution since there are several conditions and disease states, that independent of iron status, are associated with a high SF such as simple infections, liver disease, alcohol use etc. The finding of an effective regulation of iron absorption, and thus that the amounts of iron in stores are well controlled is a strong argument against a direct, simple translation of SF into iron stores in the general population. It has been suggested from high SF in some studies that high iron stores are common in man and associated with increased risks of coronary heart disease (53) and cancer (54).

However, the only associations found are with very high SF $(>200 \mu \mathrm{g} / \mathrm{L})$ in a few studies while other studies found no such relation. There is thus no reasons to believe that high iron stores are included in these associations. Actually in the most extensive material of patients with iron overload (hereditary hemochromatosis) followed over a long time, no increased incidence of coronary heart disease or cancer (disregarding the hepatocellular cancer directly caused by the iron overload) has been observed (J. Halliday personal communication). If the balance of evidence suggests a true association between the diseases mentioned and high SF it may well be in the opposite direction, that is, that the diseases or its cause for some unknown reason might lead to increased levels of serum ferritin.

\section{Iron balance in states of iron deficiency}

In a recent paper (3) we found that the relationship between iron absorption and iron stores observed in iron replete subjects, paradoxically was also valid in iron deficient subjects, i.e. in subjects with no iron stores, suggested by SF below $15 \mu \mathrm{g} / \mathrm{L}$, and with reduced amounts of circulating hemoglobin iron. This observation implied that the well established relationship between iron absorption and iron stores continued into a region of "negative values of iron stores", i.e. when iron stores were completely depleted and instead there was a lack of circulating hemoglobin iron. In this latter situation, basal iron losses are assumed to be reduced since half of the basal iron losses have been related to "physiological" blood losses, mainly from the gastrointestinal tract
$(33,55)$. With reduced hemoglobin levels these basal losses will be proportionally reduced. In the same way menstrual iron losses are also expected to be reduced in proportion to the reduction in the hemoglobin level. Recently, balance equations have been derived also for this situation indicating that iron stores and hemoglobin iron deficits form a continuum influencing iron absorption (3). It has then been possible to estimate the hemoglobin iron deficit that corresponds to a specific low bioavailability of the dietary iron and/or specific high iron requirements. When analyzing the effects expected on iron balance of a diet with a very low bioavailability, for example, in menstruating women with average total losses in developing countries, the estimated hemoglobin iron deficit would be associated with hemoglobin levels in the range 90 $100 \mathrm{~g} / \mathrm{L}$. This range is higher than that often observed in surveys in several developing countries, in turn implying that other causes of low hemoglobin levels than solely a poor diet should also be searched for. Obvious examples would be combinations of a poor diet with hookworm infestations or an inadequate supply or intake of iron supplements during pregnancies.

\section{Regulation of iron absorption}

When losses of iron for some reason exceed the absorption of iron, the negative iron balance will successively empty the iron stores. Iron absorption will then successively increase. Recent observations show that if iron balance continues to be negative, a new iron balance state will be reached by reducing the circulating hemoglobin iron. The reason is our recent, unexpected observation that iron absorption then further increases and thus beyond the point where iron stores are completely depleted (3). This means that it is not the empty iron stores per se that control the dietary iron absorption. Traditionally, two factors have been considered to control the absorption of iron: the size of the iron stores and the degree of erythropoiesis (55). When a negative iron balance has emptied the iron stores and led to a hemoglobin iron deficit, erythropoiesis will not increase in spite of the reduction in the concentration of $\mathrm{Hb}$, due to a lack of iron. This has been well demonstrated experimentally in studies in man (56).

The present new observations (3) thus imply that it is not just the empty iron stores but also the hemoglobin iron deficit that, in some probably common way, control the absorption of dietary iron. As discussed in detail in our recent paper, analyzing the physiological control of iron 
absorption and the effects of various hematological disorders on iron absorption, the balance of evidence suggests that the hepatocyte is the most probable cell that has the potential to register both the amounts of stored iron and the hemoglobin iron deficit. Much is known today about the molecular structures controlling iron absorption in the intestinal mucosal cells. The balance of evidence suggests that there is an internal signal expressing the amounts of stored iron and the hemoglobin iron deficit and that this signal to the mucosal molecular structures, controlling iron absorption, probably originates in the hepatocyte. The nature of this hypothetical signal is still unknown.

\section{REFERENCES}

1. Hallberg L, Hulthén L: Prediction of dietary iron absorption. An algorithm to calculate absorption and bioavailability of dietary iron. Am J Clin Nutr 2000;71:1147-60.

2. Hallberg L, Hulthén L, Garby L: Iron stores in man in relation to diet and iron requirements. Eur J Clin Nutr 1998:52;623-31.

3. Hallberg L, Hulthén L, Garby L: Iron stores and haemoglobin iron deficits in menstruating women. Calculations based on variations in iron requirements and bioavailability of dietary iron. Eur J Clin Nutr 2000:54;650-7.

4. Hallberg L, Björn-Rasmussen E: Determination of iron absorption from whole diet. A new twopool model using two radioiron isotopes given as haem and non-haem iron. Scand J Haematol 1972: 9;193-197.

5. Cook JD et al: Food iron absorption measured by an extrinsic tag. J Clin Invest 1972; 51;805-15.

6. Layrisse M, Martinez-Torres C: Model for measuring dietary absorption of heme iron: test with a complete meal. Am J Clin Nutr 1972: 25;401-11.

7. Björn-Rasmussen E, Hallberg L, Walker RB: Food iron absorption in man. I. Isotopic exchange between food iron and inorganic iron salt added to food: studies on maize, wheat, and eggs. Am J Clin Nutr 1972:25;317-23

8. Hallberg L: Bioavailability of dietary iron in man. Annu Rev Nutr 1981:1;123-47.

9. Hallberg L: The pool concept in food iron absorption and some of its implications. Proc Nutr Soc 1974:33;285-91

10. Baynes RD et al: Relationship between absorption of inorganic and food iron in field studies. Ann Nutr Metab 1987:31:109-16.

11. Brune M, Rossander L, Hallberg L: Iron absorption and phenolic compounds: Importance of different phenolic structures. Eur J Clin Nutr 1989:43;547-58.

12. Brune L, Rossander L, Hallberg L: Iron absorption: no intestinal adaptation to a high-phytate diet. Am J Clin Nutr 1989:49;542-5.

13. Brune M, Hallberg L, Skånberg A-B: Determination of iron-binding phenolic groups in foods. J Food Sci 1991:56;131-67.

14. Cook JD, Dassenko SA, Whittaker P: Calcium supplementation: effect on iron absorption. Am J Clin Nutr 1991:53;106-11.

15. Cook $\mathbf{J}$ et al: The effect of high ascorbic acid supplementation on body iron stores. Blood 1984:64;721-6.

16. Derman DP et al: Importance of ascorbic acid in the absorption of iron from infant foods. Scand J Haematol 1981:25;193-201.

17. Engelmann MD et al: The influence of meat on nonheme iron absorption in infnts. Pediatr Res 1998:43:768-73.

18. Hallberg L, Brune M, Rossander L: Iron absorption in man: ascorbic acid and dose-dependent inhibition by phytate. Am J Clin Nutr 1989:49; $140-4$.

19. Hallberg L et al: Calcium: effect of differen amounts on nonheme-and heme-iron absorption

\section{Concluding comments}

The body has ingenious mechanisms to prevent the development of iron deficiency and iron overload. Despite this, iron deficiency is our most common deficiency disorder worldwide. Considering the remarkable control mechanisms available in the body this is a paradoxical situation. A reasonable explanation may be the marked rather sudden increase in world population in the most recent milleniums and the accompanying change in food pattern, with reduced intake of protein and minerals, such as iron.

We are faced with important challenges to prevent iron deficiency, especially in infants, children and adolescents and in

in man. Am J Clin Nutr 1991:53;112-9.

20. Hallberg L et al: Calcium and iron absorption: mechanism of action and nutritional importance. Eur J Clin Nutr 1992:46;317-27.

21. Hallberg $\mathrm{L}$ et al: Iron absorption from bread in humans: Inhibiting effects of cereal fiber, phytate and inositol phosphates with different numbers of phospate groups. J Nutr 1992: 122;442-9.

22. Hallberg L et al: Inhibition of haem-iron absorption in man by calcium. Brit J Nutr 1992:69; 53340

23. Magnusson B et al: Iron absorption in relation to iron status. Model proposed to express results of food iron absorption measurements. Scand J Haematol 1981:27;201-8.

24. Rossander-Hulthén L, Hallberg L: Dietary factors influencing iron absorption - an overview. In: Iron nutrition in health and disease. Hallberg L, Asp NG. (eds). John Libbey: London 1996: 105-5.

25. Rossander-Hulthén L, Gleerup A, Hallberg L: Inhibitory effects of oat products on non-haem iron absorption in man. Eur J Clin Nutr 1990: 44;783-91.

26. Taylor $\mathrm{P}$ et al: Relationships among iron absorption, percent saturation of plasma transferrin and serum ferritin concentration in humans. J Nutr 1988:118;1110-5.

27. Tuntawiroon, $\mathrm{M}$ et al: Rice and iron absorption in man. Am J Clin Nutr 1990:44;489-97.

28. Tuntawiroon $\mathrm{M}$ et al: Dose-dependent inhibitory effect of phenolic compounds in foods on nonheme-iron absorption in man. Am J Clin Nutr 1991:53;554-7.

29. Siegenberg D et al: Ascorbic acid prevents the dose-dependent inhibitory effects of polyphenols and phytates on nonheme-iron absorption. Am J Clin Nutr 1991:53;537-41.

30. Gleerup A et al: Iron absorption from the whole diet: Comparison of the effect of two different distributions of daily calcium intake. Am J Clin Nutr 1995:61;97-104.

31. Hulthén L et al: Iron absorption from the whole diet. Relation to meal composition, iron requirements and iron stores. Eur J Clin Nutr 1995: 49;794-808.

32. Hallberg L, Hulthén L, Gramatkovski E: Iron absorption from the whole diet in men: how effective is the regulation of iron absorption? Am J Clin Nutr 1997:66;347-56.

33. Green R et al: Body iron excretion in man. A colloborative study. Amer J Med 1968;336-53.

34. Hallberg L, Nilsson L: Constancy of individual menstrual blood loss. Acta Obstet Gynaecol Scand 1964:43;352-9.

35. Hallberg Let al: Menstrual blood loss a population study. Variation at different ages and attempts to define normality. Acta Obstet Gynaecol Scand 1966:45;320-51.

36. Hallberg, L, Rossander-Hulthén L: Iron requirements in menstruating women. Am J Clin Nutr 1991:54;1047-58.

37. Brune M et al: Iron losses in sweat. AmJ Clin Nutr 1986:43;438-43.

38. FAO/WHO: Requirements of vitamin A, iron folate and vitamin $B_{12}$ Vol. Food and Nutrition women of childbearing age and especially during pregnancy. With the lower energy requirements of present day lifestyle, more impact must be put on a proper selection of foods, adequate composition of meals and diets to ensure a good nutrition. The weaning period with its extreme iron requirements is especially critical. More knowledge and better tools to evaluate the diet and its bioavailability are expected to improve iron nutrition.

\section{ACKNOWLEDGEMENT}

The present studies were sponsored by: The Swedish Medical Research Council (project B96-19X04721-21A), the Swedish Council for Forestry and Agriculture Research (50.0120/95 and 997/ $881,113: 3)$, the Swedish Dairy Association and the Danone International Prize for Nutrition 1999.

\section{Series Rome: FAO 23. 1988}

39. Rossander-Hulthén L, Hallberg L: Prevalence of iron deficiency in adolescents. In: Iron nutrition in health and disease. Hallberg L, Asp N-G. (eds). John Libbey: London 1996:149-56.

40. Monsen ER et al: Estimation of available dietary iron. Am J Clin Nutr 1978:31;134-41.

41. Monsen ER, Balintfy JL: Calculating dietary iron bioavailability, refinement and computerization. J Am Diet Assoc 1982:80;307-11

42. Cook JD, Dassenko SA, Lynch SR:Assessment of the role of non heme- iron availability in iron balance. Am J Clin Nutr 1991:54;717-22.

43. Tseng $\mathbf{M}$ et al: Adjustment for iron intake for dietary enhancers and inhibitors in population studies: Bioavailable iron in rural and urban residing Russian women and children. J Nut 1997:127;1456-68.

44. Bezwoda W et al: The relationship between marrow iron stores, plasma ferritin concentrations and iron absorption. Scand J Haematol 1979: 22;113-20.

45. Cook JD et al: Serum ferritin as a measure of iron stores in normal subjects. Am J Clin Nutr 1974:27;681-7.

46. Skikne BS, Flowers CH, Cook JD: Serum transferrin receptor: A quantitative measure of tissue iron deficiency. Blood 1990:75;1870-6.

47. Charlton RW et al: Hepatic storage concentrations in different population groups. Am J Clin Nut 1970:23;358-71.

48. Sturgeon, $\mathrm{P}$, Shoden A: Total liver storage iron in normal populations of the USA. Am J Clin Nutr 1971:24;469-74.

49. Pilch SM, Senti FRE: Assessment of the iron nutritional status of the US population based on data collected in the second National Health and Nutrition Examination Survey 1976-1980. Prepared for the Food and Drug Administration under Contract no FDA 223-83-2384. 1984 Bethesda, MD: Life Sciences Research Office, Federation of American Societies for Experimental Biology.

50. Hallberg Let al: Screening for iron deficiency: an analysis based on bone-marrow examinations and serum ferritin determinations in a population sample of women. Brit J Haematol 1993:85;78798.

51. Hallberg L, Björn-Rasmussen E, Jungner I: Prevalence of hereditary haemochromatosis in two Swedish urban areas. J Intern Med 1989:225; 249 55.

52. Lindmark B, Eriksson S: Regional differences in the idiopathic hemochromatosis gene frequency in Sweden. Acta Med Scand 1985: 218;299-304.

53. Salonen JT et al: High stored iron levels are associated with excess risk of myocardia infarction in eastern finnish men. Circulation 1992:86;803-11.

54. Stevens RG et al: Body iron stores and the risk of cancer. New Engl J Med 1988:319;1047-52

55. Bothwell TH et al: Iron metabolism in man Oxford: Blackwell Scientific Publications 1979.

56. Norrby A: Iron absorption studies in iron deficiency. Scand J Haematol 1974:Suppl 20;1-125. 